\title{
THE PROJECTIVE RESOLUTION OF A COMPACT SPACE ${ }^{1}$
}

\author{
ANTHONY W. HAGER ${ }^{2}$
}

\begin{abstract}
A new proof is given of Gleason's Theorem that in the category of compact Hausdorff spaces and continuous maps, each space has an essentially unique (minimal) projective resolution.
\end{abstract}

The method is a simple one, to establish by Zorn's lemma that in the family of irreducible pre-images of a given space there is a maximal member, and that this maximal member is projective. To accomplish the latter, it is convenient to use the criterion for projectivity in (b) of Theorem 1 below. The short proof of this preliminary result contains the principal points of contact between our proof of the existence of projective resolutions and those of Gleason [G] and Rainwater [R]; this is discussed below.

After this note was submitted, B. Banaschewski pointed out to me that the central idea had occurred to him some time ago, that a sketch of the consequences had been published in 1967 [ $\left.\mathrm{B}_{1}\right]$ though without much indication of the method, and that the details appear in a long paper to be published shortly $\left[\mathrm{B}_{2}\right]$. Moreover, the context of the latter is much more general than the present one. Remark 2, below, contains a discussion of the similarities and differences in the two approaches.

All spaces will be compact Hausdorff and all maps continuous. By a projective in this category is meant a space $P$ such that whenever $\alpha: P \rightarrow A$ and $\gamma: B \rightarrow A$ (onto) then there is $\varphi: P \rightarrow B$ with $\gamma \varphi=\alpha$. A projective resolution of the space $X$ is a pair $(P, \tau)$ with $P$ projective and $\tau: P \rightarrow X$ irreducible, i.e., mapping proper closed sets onto proper subsets.

TheOREM 1. The following properties of a space $P$ are equivalent.

(a) $P$ is projective.

Received by the editors November 9, 1969.

AMS 1969 subject classifications. Primary 5420, 5425, 5452, 5453; Secondary 1800.

Key words and phrases. Projective resolution, compact spaces, Gleason space, absolute.

1 This research has been supported in part by the National Science Foundation under grant GP-18825.

2 I am pleased to thank W. W. Comfort, M. Paul, Z. Tzeng, and the referee for their valuable comments on this paper. 
(b) Each irreducible map onto $P$ is a homeomorphism.

(c) Each mapping onto $P$ is a retraction, i.e., a homeomorphism of a subspace onto $P$.

Proof. Assume (a), and let $f: Y \rightarrow P$ be irreducible. There is $\varphi: P \rightarrow Y$ with $f \varphi=$ the identity on $P$. Because $f$ is irreducible, $\varphi$ is onto. Thus $f$ is one-to-one, and a homeomorphism.

Assume (b), and let $f: Y \rightarrow P$. By Zorn's lemma, there is a closed subset $S$ of $Y$ which is minimal among closed subspaces which map onto $P$. Thus $f \mid S$ is irreducible, and a homeomorphism.

Assume (c), and let $\alpha: P \rightarrow A$ and $\gamma: B \rightarrow A$. Let $Y \equiv\{(p, b): \alpha(p)$ $=\gamma(b)\}$, a closed subspace of $P \times B$. Because $\gamma$ is onto, $\pi_{P}$ (projection on $P$ ) maps $Y$ onto $P$, and by (c) there is a subspace $S$ of $Y$ with $\pi_{P} \mid S$ a homeomorphism. Then $\pi_{B}\left(\pi_{P} \mid S\right)^{-1}: P \rightarrow B$ is the desired map.

REMARK 1. This result, and its proof, are known, of course. In [G], Gleason proves successively that projectives are extremally disconnected (each open set has open closure), that each extremally disconnected space has property (b) above, that spaces with property (b) are projective. The last is not stated explicitly, but is clear from the proof of $[G, 2.5]$, a portion of which is identical to the proof (b) $\Rightarrow$ (c) $\Rightarrow$ (a) above.

That $(\mathrm{a}) \Leftrightarrow(\mathrm{c})$ is noted explicitly by Mioduszewski and Rudolph [MR, 2.1]. Their proof is that given above (though the triple $\left(Y, \pi_{P}\left|Y, \pi_{B}\right| Y\right)$ is called a "uniformization" (or "pullback") of the pair $\alpha, \gamma$, and its existence is asserted on category-theoretic grounds).

The "minimal subspace lemma" in (b) $\Rightarrow(c)$ above is used by both Gleason and Rainwater.

We turn to projective resolutions. It is necessary to do a little preliminary book-keeping in the vein of [K, p. 151].

Let $X$ be a fixed space. In the family of pairs $(A, \alpha)$ where $\alpha: A \rightarrow X$ is irreducible, define $(A, \alpha) \geqq(B, \gamma)$ if there is a map $f_{\alpha \gamma}: A \rightarrow B$ with $\gamma f_{\alpha \gamma}=\alpha$. Define $(A, \alpha) \equiv(B, \gamma)$ if $(A, \alpha) \geqq(B, \gamma)$ and $(B, \gamma) \geqq(A, \alpha)$. Call $(A, \alpha)$ a maximal pair if $(B, \gamma) \geqq(A, \alpha)$ implies $(B, \gamma) \equiv(A, \alpha)$.

(i) $(A, \alpha) \equiv(B, \gamma)$ if and only if there is a homeomorphism $h: A \rightarrow B$ with $\gamma h=\alpha$.

(ii) The maximal pairs are exactly the projective resolutions of $X$.

To prove these, we need the following.

Lemma. Let $\gamma: B \rightarrow X$ and one of $f, g: A \rightarrow B$ be irreducible. If $\gamma f=\gamma g$, then $f=g$.

Proof. If $f(a) \neq g(a)$ for an $a \in A$, then choose disjoint open neighborhoods $U$ and $V$, of $f(a)$ and $g(a)$. If $f$ is irreducible, then 
$f\left(f^{-1}(U) \cap g^{-1}(V)\right)$ contains an open set $G$ (as is quickly checked). Then $\gamma(B-G)=X$, contradicting irreducibility of $\gamma$.

Observe that this Lemma implies the uniqueness of the connecting maps $f_{\alpha \gamma}$ defined above. This will be used in the proof of Gleason's Theorem below.

To prove (i) and (ii), we use this immediate consequence of the Lemma: If $\gamma: A \rightarrow X$ is irreducible and $g: A \rightarrow A$ has $\gamma g=\gamma$, then $g$ is the identity on $A$. (This statement is implicit in [R, p. 735].)

To prove (i): If $h$ is such a homeomorphism, let $f_{\alpha \gamma}=h$ and $f_{\gamma \alpha}=h^{-1}$. Conversely, if $(A, \alpha) \equiv(B, \gamma)$, then by the Lemma, $f_{\alpha \gamma} f_{\gamma \alpha}$ and $f_{\gamma \alpha} f_{\alpha \gamma}$ are the identities on $A$ and $B$, respectively, so $f_{\alpha \gamma}=f_{\gamma \alpha}^{-1}$ and we set $h=f_{\alpha \gamma}$.

To prove (ii): If $(P, \tau)$ is a projective resolution, then given $(B, \gamma)$, projectivity of $P$ yields $(P, \tau) \geqq(B, \gamma)$. So if to begin with, $(B, \gamma)$ $\geqq(P, \tau),(B, \gamma) \equiv(P, \tau)$ follows. Conversely, let $(P, \tau)$ be a maximal pair. We shall verify (b) of Theorem 1 . Let $g: A \rightarrow P$ be irreducible. Let $\alpha=\tau g$. Clearly, $\alpha$ is irreducible and $(A, \alpha) \geqq(P, \tau)$. By maximality, $(A, \alpha) \equiv(P, \tau)$; so by (i) there is a homeomorphism $h: P \rightarrow A$ with $\alpha h=\tau$. Evidently, $\alpha(h g)=\alpha$, so by the Lemma, $h g$ is the identity on $A$. Then $g=h^{-1}$ is a homeomorphism, as desired.

THEOREM 2 (GLEASON). Each space has a projective resolution. If $(P, \tau)$ and $\left(P^{\prime}, \tau^{\prime}\right)^{\cdot}$ are projective resolutions of the same space, then there is a homeomorphism $h: P \rightarrow P^{\prime}$ with $\tau^{\prime} h=\tau$.

Proof. For the uniqueness: if $(P, \tau)$ and $\left(P^{\prime}, \tau^{\prime}\right)$ are projective resolutions of $X$, then projectivity of $P$ implies $(P, \tau) \geqq\left(P^{\prime}, \tau^{\prime}\right)$, and likewise, $\left(P^{\prime}, \tau^{\prime}\right) \geqq(P, \tau)$; so $(P, \tau) \equiv\left(P^{\prime}, \tau^{\prime}\right)$, and (i) yields the desired homeomorphism.

For the existence, we shall find a "maximal pair" and apply (ii).

Let $D$ be discrete $X$, and $\beta D$ the Stone-Cech compactification. Let $\beta$ be the set of pairs $(A, \alpha)$ for which $A$ is a quotient of $\beta D$-an actual set of equivalence classes in $\beta D$. We claim that:

$\left({ }^{*}\right)$ given a pair $(B, \gamma)$, there is $(A, \alpha) \in \odot$ with $(A, \alpha) \equiv(B, \gamma)$. To see this, observe that $\beta D$ is projective-this is trivial to verify. So, with $\nu: \beta D \rightarrow X$ the extension of the identity on $X$ over $\beta D$, there is $\varphi: \beta D \rightarrow B$ with $\gamma \varphi=\nu$. One checks that $\varphi$ is onto, and the desired member of $\beta$ is obvious.

Now, $\odot$ together with $\geqq$ is a partially ordered set (according to the definition in $[\mathbf{K}]: \geqq$ is a transitive relation). We shall show that $P$ has a maximal member. Using $\left({ }^{*}\right)$ such a maximal member is a "maximal pair."

Let $\mathcal{C}$ be a chain in $P$. Construct an inverse system by equipping 
the family $\{A:(A, \alpha) \in \mathfrak{C}\}$ with the maps $f_{\alpha \gamma}: A \rightarrow B$ with $\gamma f_{\alpha \gamma}=\alpha$, when $(A, \alpha) \geqq(B, \gamma)$ in $\mathcal{C}$. This is possible because the connecting maps are unique. Let $I$ be the inverse limit (the subset of $\prod\{A:(A, \alpha) \in \mathbb{C}\}$ of points $\left(p_{(A, \alpha)}\right)$ such that when $(A, \alpha) \geqq(B, \gamma)$ then $\left.f_{\alpha \gamma}\left(p_{(A, \alpha)}\right)=p_{(B, \gamma)}\right)$. Define $\iota: I \rightarrow X$ by picking $(A, \alpha) \in \mathcal{C}$, and letting $\iota=\alpha \pi_{A}$, where $\pi_{A}$ is the projection: $I \rightarrow A$. By definition of the inverse limit, $\alpha \pi_{A}=\gamma \pi_{B}$ for any other $(B, \gamma) \in \mathcal{C}$, and $\iota$ is well-defined. We shall show that $\iota$ is irreducible. Then, the equation $\iota=\alpha \pi_{A}$ shows that $(I, \iota) \geqq(A, \alpha)$. Using $\left({ }^{*}\right)$, choose $\left(I^{\prime}, \iota^{\prime}\right)$ in $\odot$ with $\left(I^{\prime}, \iota^{\prime}\right) \equiv(I, \iota)$. Evidently, $\left(I^{\prime}, \iota^{\prime}\right)$ is the required upper bound for $\mathcal{C}$. So, let $F$ be a proper closed subset of $I$. We may as well assume that $F=I-G$ where $G$ is basic, of the form $I \cap\left(\pi_{\left(A_{1}, \alpha_{1}\right)}^{-1}\left(G_{1}\right) \cap \cdots \cap \pi_{\left(A_{n}, \alpha_{n}\right)}^{-1}\left(G_{n}\right)\right)$, , where $G_{i}$ is open in $A_{i}$. Since $\mathrm{e}$ is a chain, $\left\{\left(A_{i}, \alpha_{i}\right)\right\}_{i=1}^{n}$ has a maximum, say $(A, \alpha)$, and using the definition of the inverse limit, $G$ collapses to $I \cap \pi_{(A, \alpha)}^{-1}(H)$ for a suitable open $H \subset A$. Then,

$$
\iota(F)=\alpha \pi_{A}(F)=\alpha \pi_{A}(I-G)=\alpha(A-H) .
$$

Because $\alpha$ is irreducible, $\alpha(A-H) \neq I$. So $\iota$ is irreducible, chains in $P$ are upper bounded, and Zorn's lemma gives a maximal member $(P, \tau)$ of $P$.

REMARK 2. In $\left[\mathrm{B}_{2}\right]$, Banaschewski considers a category together with a class of its morphisms-call the class $\mathfrak{N}$-which together satisfy five axioms, defines $\Re$-projectivity and $\Re$-projective resolution in the category, and proves, by what is essentially the same method as is used here, that the $\mathfrak{T}$-projective resolution exists for each object which satisfies a pair of conditions concerning its "essential covers" - here these are the irreducible pre-images. This pair of conditions is used to the same effect as we have used $\beta D$ in the proof of Theorem 2, namely to guarantee an appropriate set in which to use Zorn's lemma. One of Banaschewski's axioms asserts that well-ordered inverse systems have upper bounds; using Zorn's lemma, he chooses a maximal inverse system "starting at the given object"; the stipulated upper bound is shown to be projective via [ $\mathbf{B}_{2}$, Proposition 1]-quite the same as our Theorem 1 , but more general. The proof of $\left[\mathbf{B}_{2}\right.$, Proposition 1] also is much the same as the proof of Theorem 1, requiring the existence of pullbacks, which is another of the axioms. Thus, at this point in the development, Banaschewski does not use the "minimal subspace lemma" referred to in Remark 1, but needs it later in verifying that certain topological categories satisfy the axioms. The actual applications of $\left[\mathrm{B}_{2}\right.$, Proposition 1] and our Theorem 1 differ a bit in detail. Ours proceeds through (ii) above, and hence through the Lemma used in the proof of (ii). This Lemma asserts what 
Banaschewski calls the rigidity of the projective resolution, an idea which he discusses much later.

REMARK 3. There are other constructions of the projective resolution besides those due to Gleason and Rainwater. Among the moreor-less direct ones, see $\left[\mathrm{P}_{1}\right]$ (and $\left[\mathrm{P}_{2}\right]$ ), $[\mathrm{I}]$ (and $[\mathrm{IF}]$ ), and $[\mathrm{W}]$. Less direct constructions appear in [FGL] and [S].

Projectivity in other topological categories is treated in $\left[\mathrm{B}_{2}\right],[\mathbf{F}]$, $[\mathrm{MR}]$, and [St] (as well as [G]).

The papers $\left[\mathbf{B}_{2}\right],[\mathrm{IF}]$ and $\left[\mathrm{P}_{3}\right]$ contain excellent bibliographies on the general subject.

\section{REFERENCES}

$\left[\mathbf{B}_{1}\right]$. B. Banaschewski, Projective covers in certain categories of topological spaces, Proc. Second Prague Topological Sympos. 1966, Academia, Prague, 1967, pp. 52-55. MR 38 \#654.

$\left[\mathbf{B}_{2}\right]$. - Projective covers in categories of topological spaces and topological algebras (to appear).

[FGL]. N. J. Fine, L. Gillman and J. Lambek, Rings of quotients of rings of functions, McGill Univ. Press, Montreal, Que., 1966. MR 34 \#635.

[G]. Andrew M. Gleason, Projective topological spaces, Illinois J. Math. 2 (1958), 482-489. MR 22 \#12509.

[I]. S. Iliadis, Absolutes of Hausdorff spaces, Dokl. Akad. Nauk SSSR 149 (1963), 22-25 = Soviet Math. Dokl. 4 (1963), 295-298. MR 28 \#589a.

[IF]. S. Iliadis and S. Fomin, The method of concentric systems in the theory of topological spaces, Uspehi Mat. Nauk 21 (1966), no. 4 (130), 47-76= Russian Math. Surveys 21 (1966), no. 4, 37-62. MR 34 \#3526. 1136.

[K]. J. L. Kelley, General topology, Van Nostrand, Princeton, N. J., 1955. MR 16,

[MR]. J. Mioduszewski and L. Rudolph, On projective spaces and resolutions in categories of completely regular spaces, Colloq. Math. 18 (1967), 185-196. MR 37 \#6915.

$\left[\mathrm{P}_{1}\right]$. V. Ponomarev, Normal spaces as images of zero-dimensional ones, Dokl. Akad. Nauk SSSR 132 (1960), 1269-1272 =Soviet Math. Dokl. 1 (1960), 774-777. MR 23 \#A622.

$\left[\mathbf{P}_{2}\right] .-$ On the absolute of a topological space, Dokl. Akad. Nauk SSSR 149 (1963), 26-29 =Soviet Math. Dokl. 4 (1963), 299-302. MR 28 \#589b.

$\left[\mathbf{P}_{3}\right] . \longrightarrow$, Spaces co-absolute with metric spaces, Uspehi Mat. Nauk 21 (1966), no. 4 (130), 101-132 = Russian Math. Surveys 21 (1966), no. 4, 87-113. MR 34 \#788.

$[\mathbf{R}]$. John Rainwater, $A$ note on projective resolutions, Proc. Amer. Math. Soc. 10 (1959), 734-735. MR 23 \#A618.

[Se]. Z. Semadeni, Spaces of continuous functions on compact sets, Advances in Math. 1 (1965), fasc. 3, 319-382. MR 32 \#2937.

[St]. D. P. Strauss, Extremally disconnected spaces, Proc. Amer. Math. Soc. 18 (1967), 305-309. MR 35 \#961.

[W]. E. C. Weinberg, Higher degrees of distributivity in lattices of continuous functions, Thesis, Purdue University, Lafayette, Ind., 1961.

Wesleyan University, Middletown, Connecticut 06457 